\title{
Singular Phenomena in Kinematics
}

\author{
P. S. Donelan and C. G. Gibson
}

\begin{abstract}
Les principes généraux de la theorie des systèmes articulés n'ont pas encore été éclairais et on est reduit à un ensemble de recherches isolées et de résultats curieux sans liens apparents entre eux. C'est une raison de plus pour engager les géomètres à éclairer cette question encore obscure: les progrès de la science se font parfois par les côtés les plus inattendus. Gabriel Koenigs, Leçons de Cinématique.
\end{abstract}

\section{Introduction}

The kinematics of rigid bodies has been an essential ingredient of engineering for millenia. The connections between engineering applications and mathematics date back at least as far as Archimedes and almost certainly further. However the serious mathematization of the subject, in particular the application of algebraic (in addition to geometric) methods began in the 18th century and received its strongest impetus from the Industrial Revolution. Mechanisms for converting circular into rectilinear motion were widely adopted in industrial machinery. For many purposes approximate linear motion is an acceptable substitute for exact linear motion. A well known example is the Watt 4 -bar of Fig. 1 discovered in 1784. The device comprises three smoothly jointed bars moving with one degree of freedom (dof), the mid-point of the middle (or coupler) bar describing the Watt curve of Fig. 1. The curve has a self-crossing with two branches, one having 5-point contact with its tangent at that point, so being an excellent approximation to a straight line.

Figure 1: The Watt 4-bar mechanism.

A central feature of curves traced by planar mechanisms is that they exhibit singularities. Generally one expects to encounter nodes, i.e. ordinary double points, as in the Watt curve above. The engineering significance of a node is that the mechanism can transport an object to a "working position", leave it there, and then retrieve it after a prescribed rotation of a crank. Nodes can degenerate to other singularities, in particular to cusps. The most familiar example is the cusp on the cycloid generated by a point on the circumference of a rolling wheel. Cusps likewise have engineering interest, namely that they provide "dwell", i.e. the property that the tracing point comes instantaneously to rest. In kinematic terms, the tracing point coincides with the instantaneous centre of rotation of the moving plane. As the mechanism moves so the instantaneous centre traces out a curve, the moving centrode. This is the first example of a bifurcation curve, representing choices of tracing points which give rise to trajectories exhibiting exceptional singular behaviour.

Watt's mechanism is a classical example of a planar linkage - it belongs to the family of planar 4-bars whose geometry is discussed in Section 2. The joints are simply revolute hinges. Spatial linkages use a greater variety of joints [35, page 8] such as the spherical or S-joint (realized in engineering by a ball joint), the revolute or $\mathrm{R}$-joint (hinge), the prismatic or $\mathrm{P}$-joint (slider) and the helical or $\mathrm{H}$-joint (screw). $\mathrm{R}-, \mathrm{P}-$ and $\mathrm{H}$-joints have one dof while $\mathrm{S}$-joints have three. A simple example is the spatial 4-bar or RRRR device, analogous to the planar 4-bar. In general such devices are rigid, for generically the dofs of the joints of a closed loop must be at least 7 for mobility. However there are exceptional cases. When the four hinges are parallel one has the planar 4-bar (when the hinges are concurrent at a point at infinity) and the spherical 4-bar (when the hinges are concurrent at a finite point). And there exist extremely interesting mobile RRRR 
devices (the Bennett mechanisms [4,5]) discovered at the turn of the century, in which the hinges fail to be concurrent, but belong to the reguli of a hyperboloid. One broad class of motions with several dofs arises on composing a number of simpler motions, yielding serial devices such as robot arms, in which the joints are either revolute or prismatic. A second approach is to apply several simultaneous constraints, to obtain parallel devices. For example, by constraining $k$ fixed points $P_{1}, \ldots, P_{k}$ on the body to move on corresponding surfaces $S_{1}, \ldots, S_{k}$ in the ambient space we obtain a motion with $6-k$ degrees of freedom. Motions with three dofs are of particular interest. The simplest case is when $S_{1}, S_{2}, S_{3}$ are planes, yielding the classical Mannheim motions. When $S_{1}, S_{2}, S_{3}$ are spheres we obtain the Remote Centre of Compliance (RCC) device [18], used in assembly applications where parts must be mated with clearance less than the accuracy of the robot.

For 1-dof spatial mechanisms, singularities do not play a major role. The underlying reason is that in 3-space curves do not in general self-intersect and the analogue of the instantaneous centre of rotation is an instantaneous screw axis, which in general is not fixed. However the RSSR linkage has two dof, so traces a surface (the qeeroid of [51]) which can exhibit cross-caps. Until relatively recently, rigid body motions with several dofs were largely of theoretical interest. However the rapid development of robot technology and computer control systems has created a surge of interest. Of special interest, both for mechanical reasons and for the development of effective control algorithms, are singularities of such motions. Thus a central problem is to analyse the trajectory singularities. This can be attacked at several levels - which singularities would one expect generically, or within a given broad class, or for a specific family? This paper is intended to survey our knowledge to date. The systematic development of the ideas of singularity theory within kinematics has its genesis in a detailed study [30] of the singularities exhibited by the planar 4-bar, and the paper [13] on generic singularities of planar Euclidean motions. The resulting "singular viewpoint" lends coherence to a number of interesting kinematical topics, and enables the classical core of the subject to be extended quite significantly.

We would like to express our thanks to Ton Marar who proposed that the second author deliver a series of lectures on Singular Phenomena in Kinematics in the Instituto de Ciências Matemáticas de São Carlos, University of São Paulo, Brazil. Those lectures form the basis of this paper. We would also like to thank the following colleagues with whom we have worked on the problems discussed here: Wendy Hawes, Cathy Hobbs, Duncan Marsh, Peter Newstead and Yanling Xiang, and to acknowledge the benefit of Bill Bruce's help on singularity theory over a number of years. Neil Kirk kindly advised us on the use of his program Transversal, and Richard Morris gave invaluable help with computer graphics.

\section{Geometry of The Planar Four-Bar}

Studying the motion of a specific family of mechanical or robotic device proceeds generally in two steps. The first is to identify a configuration space for the motion, typically a real variety whose points correspond one-to-one with the configurations of the device, and to determine the natural map from this configuration space into the Lie group of rigid displacements of the ambient space. This is the kinematic mapping of theoretical robotics, with image the kinematic image. The second step is to compose the natural action of the group on the ambient space with the motion to view the trajectories as images of the kinematic image under a family of submersions. The singular behaviour will vary with the design parameters for the family, representing the various distances and angles specifying a given device. In the space of design parameters one expects to find a 
hypersurface (for historical reasons we would like to dub it the Grashof hypersurface) giving rise to degenerate singular behaviour. In this section we give a brief account, based on [30], of the geometry of a classic example, the planar 4-bar, illustrated in Fig. 2. It comprises a fixed base to which are attached two links (or bars), each free to rotate about a point in the base, and a coupler link joining them to form a closed quadrilateral.

Figure 2: A 4-bar coupler curve.

The design parameters for the 4 -bar are the link lengths $d_{1}, d_{2}, d_{3}, d_{4}$. The configuration space can be determined in terms of the link directions, represented by unit complex numbers $z_{1}, z_{2}, z_{3}, z_{4}$. Write $z_{k}=x_{k}+i y_{k}$ with $x_{k}, y_{k}$ real for $1 \leq k \leq 4$; assuming the base to be fixed, $x_{4}=-1, y_{4}=0$. Using the closure of the quadrilateral the configuration space is represented by five equations in six real unknowns, defining an algebraic variety in $\mathbf{R}^{6}$ :

$$
\begin{aligned}
d_{1} x_{1}+d_{2} x_{2}+d_{3} x_{3} & =d_{4} \\
d_{1} y_{1}+d_{2} y_{2}+d_{3} y_{3} & =0 \\
x_{k}^{2}+y_{k}^{2} & =1 \quad(1 \leq k \leq 3) .
\end{aligned}
$$

We would like to ensure that these equations have at least one real solution. Write the link lengths in increasing order of magnitude as $e_{1}, e_{2}, e_{3}, e_{4}$ : then the required condition is that $e_{4} \leq e_{1}+e_{2}+e_{3}$. We assume henceforth that we are in this constructible case. For geometry, we complexify and projectivize to obtain the equations:

$$
\begin{aligned}
d_{1} x_{1}+d_{2} x_{2}+d_{3} x_{3} & =d_{4} w \\
d_{1} y_{1}+d_{2} y_{2}+d_{3} y_{3} & =0 \\
x_{k}^{2}+y_{k}^{2} & =w^{2} \quad(1 \leq k \leq 3),
\end{aligned}
$$

defining an algebraic variety (the linkage curve $C$ ) in complex projective 6 -space $P \mathbf{C}^{6}$. It is easy to show that $C$ meets the hyperplane at infinity $w=0$ in two complex conjugate lines $L, \bar{L}$. Singularities of $C$ are determined by a drop in rank of the $5 \times 7$ Jacobian matrix of equations (1). Computations show that the infinite singularities $(w=0)$ are at three pairs of conjugate points on $L, \bar{L}$. Finite singularities $(w \neq 0)$ occur only when $y_{1}=y_{2}=y_{3}=0$, representing the flattening of the quadrilateral: and then substitution in equation (1) yields the Grashof relations

$$
d_{1} \pm d_{2} \pm d_{3} \pm d_{4}=0
$$

Four-bars which fail to satisfy these relations are generic. Scaling the parameters so that $d_{4}=1$, the relations define eight planes (whose union is the Grashof surface) in the 3-space with coordinates $d_{1}, d_{2}, d_{3}$ of which one (all signs positive) does not correspond to a constructible mechanism. Four of the others (one sign negative) define rigid 4-bars, on the boundary of the constructible region. The remaining three planes satisfy the condition $E=e_{1}+e_{4}-e_{2}-e_{3}=0$ and intersect pairwise in lines, and mutually at the point $d_{1}=d_{2}=d_{3}=1$. That yields Table 1 below. The type symbol represents the number of finite singularities on $C$. By Bezout's Theorem $C$ has degree 8 , so the complex residual curve $R$ obtained by deleting the lines $L, \bar{L}$ from $C$ will be a curve of degree 6 . $R$ meets $w=0$ at the six infinite singular points. The global geometry of the complex curve $R$ is easily ascertained. For example, for type $\mathrm{O}, R$ is an elliptic sextic; for type I it is an irreducible rational sextic with a single node, and so on.

The real curve $R$ is the configuration space for the planar 4 -bar, in the sense that its points correspond one-to-one with the configurations of the moving quadrilateral. In fact, there is a family of configuration spaces parametrized by the design parameters which can be thought of as 


\begin{tabular}{|c|c|l|}
\hline type & relation & name \\
\hline \hline $\mathrm{O}$ & $e_{1}+e_{4} \neq e_{2}+e_{3}$ & generic \\
$\mathrm{I}$ & $e_{1}+e_{4}=e_{2}+e_{3}$ & circumscriptible \\
$\mathrm{I}^{\prime}$ & $e_{1}+e_{2}+e_{3}=e_{4}$ & rigid \\
$\mathrm{II}$ & $e_{1}=e_{2} \neq e_{3}=e_{4}$ & parallelogram \\
$\mathrm{III}$ & $e_{1}=e_{2}=e_{3}=e_{4}$ & rhombus \\
\hline
\end{tabular}

Table 1: Five basic types of 4-bar.

a single big configuration space, and a key question is how the geometry of the configuration space changes within the family. Basic degenerations take place on the Grashof surface. For generic 4-bars the configuration space $R$ is a smooth compact 1-manifold: for $E>0$ it is topologically a circle and for $E<0$ a union of two circles. In fact the connected components of the complement of the Grashof surface provides further refinement, enumerated originally by Hain [31]. They classify 4-bars in terms of the 'rocking' or 'cranking' capabilities of the input/output links, in particular whether they rock 'inwards' or 'outwards'. More generally, the Grashof hypersurface associated to the planar $n$-bar loop reduces (as in the case $n=4$ ) to a union of hyperplanes. The topology of the configuration space for more general mechanisms has been studied by Niemann, in his unpublished thesis [47], via a natural Morse function on the big configuration space, yielding an explicit formula for the Betti numbers: for $n=5$ the configuration space is a compact surface of genus $\leq 4$, and for $n=6$ it is a compact $3-$ manifold with a finitely presented fundamental group.

The second step in the process is to examine trajectories associated to the mechanism. The coupler bar of the 4-bar determines the moving plane, and any choice of tracing point $P$ in it will trace a trajectory, in the fixed plane. The geometry of a trajectory can be elucidated by considering it as a projection of the residual curve $R$. Write $P=d_{1} z_{1}+k z_{2}$ for some complex number $k=k_{1}+i k_{2}$ with $k_{1}, k_{2}$ real, and think of $P$ having homogeneous coordinates $p=\left(p_{1}, p_{2}, p_{3}\right)$ where

$$
p_{1}=d_{1} x_{1}-k_{2} y_{2}+k_{1} x_{2}, \quad p_{2}=d_{1} y_{1}-k_{2} x_{2}+k_{1} y_{2}, \quad p_{3}=w .
$$

These formulas define a family of projections $\pi_{k}: \mathrm{PC}^{6}-V \rightarrow \mathrm{PC}^{2}$ with $V$ the centre of projection, i.e. the 3-space defined by the vanishing of $p_{1}, p_{2}, p_{3}$. Under this projection $L, \bar{L}$ map to the circular points at infinity $I=(1: i: 0), \bar{I}=(1:-i: 0)$ and the image of the restriction $\pi_{k} \mid R$ is an algebraic curve $C_{k}$ in $\mathrm{PC}^{2}$, the complex coupler curve.

To understand the projection better, write $W$ for the 4 -space in $\mathrm{PC}^{6}$ defined by the linear equations in (1). $W$ intersects the centre of projection $V$ in a line $L_{1}$ which (bar exceptional cases) does not meet $R$. As $R \subseteq W$ it suffices to consider the projections $\pi_{k}: W-L_{1} \rightarrow \mathrm{PC}^{2}$ given by the same forms $p_{1}, p_{2}, p_{3}$. The key observation is that $L_{1}$ meets both $L$ and $\bar{L}$. This has the following consequence. Write $q_{1}, q_{2}, q_{3}$ for the quadrics in $W$ obtained by intersecting $W$ with $x_{1}^{2}+y_{1}^{2}=w^{2}$, $x_{2}^{2}+y_{2}^{2}=w^{2}, x_{3}^{2}+y_{3}^{2}=w^{2}$ and consider the resulting net of quadrics $\lambda_{1} q_{1}+\lambda_{2} q_{2}+\lambda_{3} q_{3}$. Given a point in $W$, the condition for a quadric in the net to pass through that point is a linear one in $\lambda_{1}, \lambda_{2}, \lambda_{3}$ defining a pencil of quadrics in $W$ : taking the given point to be any point of $L_{1}$ not on $L, \bar{L}$ we see that a quadric in the pencil meets $L_{1}$ in three points, hence contains $L_{1}$. There is therefore a pencil of quadrics in the net containing $L_{1}$. The intersection of the pencil is a Segre quartic surface $S$. Provided the pencil is generic (the tracing points for which the pencil fails to be generic form a "bad" curve in the moving plane) $S$ is known to contain sixteen lines, any one of which meets five other mutually skew lines. $L, \bar{L}$, projecting to $I, \bar{I}$, are two of the lines in $S$ 
meeting $L_{1}$, and there will be three others $L_{2}, L_{3}, L_{4}$, projecting to points $I_{2}, I_{3}, I_{4}$. In this way we obtain the classical [36] birational isomorphism between $S$, with the six lines deleted, and the plane, with the conic $F$ through $I, \bar{I}, I_{2}, I_{3}, I_{4}$ deleted. $F$ is the circle of singular foci well-known in the engineering literature [35]. In particular the isomorphism restricts to one between $R$ and $C_{k}$, ensuring that $C_{k}$ has the same geometric genus, and that off $F$ it has the same number and type of singularities as $R$ (none in the generic case). The only other singularities of $C_{k}$ are on $F: I, \bar{I}$ are ordinary triple points, and each of $I_{2}, I_{3}, I_{4}$ is either a node or a cusp. In the exceptional cases one also learns a great deal about the possible singularities on a trajectory from the geometry of the associated degenerate Segre surface [11].

To summarise, suppose we start with a generic 4 -bar. For a general choice of tracing point, $C_{k}$ has five singular points, namely two ordinary triple points at the circular points at infinity, and three ordinary double points on the circle of singular foci. Any transition from one type of mechanical behaviour to another will have to take place on a "bifurcation" curve of tracing points in the moving plane. The key aim of the singular approach is to understand such behaviour better.

\section{The Singular Viewpoint}

The example of the 4-bar mechanism shows that the singular viewpoint can be applied at two levels; these are intimately connected. At one level we may consider the motion as a whole - this can be formalised as a map from some configuration space (with possible singularities) into the group of rigid body displacements. In the case of effective robot arms the motion is typically a smooth map between a 6-dimensional configuration space and the 6-dimensional group of rigid body displacements of 3-space. There are few specific results in singularity theory about such maps but some attempts have been made to understand them in the kinematics literature [48,52]. For spatial motions with three or fewer parameters there are generically no singularities. For planar motions, however, the group is 3-dimensional and much more is known already about stable singularities. At a second level we are interested in the trajectory maps whose images are the trajectories in the ambient space of points in the moving body. We consider trajectories as maps in their own right and then establish connections with the underlying motion.

\subsection{Equivalence of Motions}

We will set up a formal framework in which these intuitions can be made precise. Let $S E(p)$ denote the special Euclidean group, i.e. the Lie group of proper rigid motions of $\mathbf{R}^{p}$ with its standard Euclidean structure. By an $n$-dof motion of $\mathbf{R}^{p}$ we mean a smooth mapping $\lambda: N \rightarrow S E(p)$, where $N$ is a smooth manifold of dimension $n$. Motions (or their germs) can be classified under $\mathcal{A}$-equivalence. At a naive level this already provides useful information: later we shall see that the $\mathcal{A}$-type of a motion germ has a significant effect on which singularities can be exhibited by trajectories. However $\mathcal{A}$-equivalence fails to take into account the group structure on the target $S E(p)$. Given that the position of a rigid body is represented by an element of $S E(p)$ only up to choices of body and space coordinates, one is led naturally to the following definition.

Definition 3.1 Let $\lambda_{1}: N_{1}, x_{1} \rightarrow S E(p), g_{1}$ and $\lambda_{2}: N_{2}, x_{2} \rightarrow S E(p), g_{2}$ be (germs of) $n$-dof motions of $\mathbf{R}^{p}$. Then $\lambda_{1}, \lambda_{2}$ are $\mathcal{I}$-equivalent (isometry equivalent) if there exist an invertible germ $\phi: N_{1}, x_{1} \rightarrow N_{2}, x_{2}$ and elements $h, k$ in $S E(p)$ such that $\lambda_{2}(x)=h . \lambda_{1}(\phi(x)) . k$. 
$\mathcal{I}$-equivalent motions are automatically $\mathcal{A}$-equivalent, but the former defines a much finer equivalence relation. One objective will be to find first order $\mathcal{I}$-invariants of motion germs, particularly in the case $p=3$ (see Section 4). $\mathcal{I}$-equivalence is implicit in the classical kinematics literature and a good deal is known about the invariants (the instantaneous invariants of [6]) in the special case of (planar and spatial) 1-dof motions. Nevertheless, the fineness of the relation means that it does not yield a tractable basis for a classification of motion germs.

\subsection{A Transversality Lemma for Trajectories}

The action of the group on $\mathbf{R}^{p}$ yields a smooth mapping $M_{\lambda}: N \times \mathbf{R}^{p} \rightarrow \mathbf{R}^{p}$ defined by $(t, w) \mapsto$ $\lambda(t)(w)$. Indeed this map may be thought of as a composite of the group action with the motion in the following sense. Let $\alpha: S E(p) \times \mathbf{R}^{p} \rightarrow \mathbf{R}^{p}$ denote the action of the group. Then $M_{\lambda}=$ $\alpha \circ(\lambda \times 1)$. For any fixed choice of $w \in \mathbf{R}^{p}$ there is a smooth mapping $M_{\lambda, w}: N \rightarrow \mathbf{R}^{p}$ defined by $t \mapsto \lambda(t)(w)$, which we refer to as the trajectory of $w$ under $\lambda$. So $M_{\lambda}$ may be thought of as a $p$-parameter family of trajectories. Given positive integers $r$ and $k$ this induces a multijet extension ${ }_{r} j^{k} M_{\lambda, w}: N^{(r)} \rightarrow{ }_{r} J^{k}\left(N, \mathbf{R}^{p}\right)$, and since $M_{\lambda, w}$ depends smoothly on $w$ there is a well defined mapping

$$
{ }_{r} j_{1}^{k} M_{\lambda}: N^{(r)} \times \mathbf{R}^{p} \longrightarrow{ }_{r} J^{k}\left(N, \mathbf{R}^{p}\right) .
$$

where the subscript 1 indicates we are taking jets with respect to the first component only. We then have the following Basic Transversality Lemma [23].

Lemma 3.1 Let $\mathcal{S}$ be a finite stratification of ${ }_{r} J^{k}\left(N, \mathbf{R}^{p}\right)$. The set of $n$-dof motions $\lambda: N \rightarrow$ $S E(p)$ with ${ }_{r} j_{1}^{k} M_{\lambda}$ transverse to $\mathcal{S}$ is residual in $C^{\infty}(N, S E(p))$, endowed with the Whitney $C^{\infty}$ topology.

We note that the form of this lemma falls within the ambit of the theorem of Montaldi on composite maps [43] since the group action is submersive, yielding a simpler proof than the original. It is important to realise that this is a result of general kinematics, i.e. it is concerned with generic motions of a rigid body. However one of the ultimate objectives is to apply the general framework to robotics, i.e. mechanically generated motions of a rigid body. From the mathematical point of view the step from kinematics to robotics is a major one; in general kinematics a motion can be rendered generic by arbitrarily small deformations within the infinite dimensional space of all motions, but in robotics the motion can only be deformed via the finite dimensional space of design parameters. Thus a serious underlying problem of the subject is to study the genericity of examples. At present there are no general results in this direction. Current research on the family of 4-bars suggests that the genericity question for a given example is a very subtle one, and that even quite simple examples can fail to be generic.

\subsection{Classification}

In the above situation there is a codimensional restriction on the relevant strata of $\mathcal{S}$, which we need to make precise. Let $X$ be an $\mathcal{A}$-invariant smooth submanifold of ${ }_{r} J^{k}(n, p)$, giving rise in a natural way to an $\mathcal{A}$-invariant smooth submanifold $Y$ of ${ }_{r} J^{k}\left(N, \mathbf{R}^{p}\right)$. Suppose that ${ }_{r} j_{1}^{k} M_{\lambda}$ is transverse to $\mathcal{S}$, and that $Y$ is one of the strata. Then a necessary condition for the pull-back of $Y$ under ${ }_{r} j_{1}^{k} M_{\lambda}$ to be non-void is that $Y$ has codimension $\leq r n+p$, the dimension of the domain. However local triviality of the multijet bundle shows that the codimensions of $X, Y$ in their respective multijet 
spaces differ by $(r-1) p$. Thus the condition on $X$ is that its codimension should be $\leq 2 p+r(n-p)$. Given $n, p$ the guiding principle for classification is to list strata in the multijet space up to this value. In particular, for a generic motion, non-stable $\mathcal{A}$-simple multigerms of trajectories will be of $\mathcal{A}_{e}$-codimension $\leq p$. For applications to robotics the important cases are $p=2$ and $p=3$ where it is realistic to expect complete solutions. For $p=2$ there is a complete listing for all dimensions $n$, with $n=1, n=2$ and $n \geq 3$ the principal subdivisions, described in Section 5 . For $p=3$ the listings are only complete for $n \leq 3$, and then only as the result of using computer algebra programs (see Section 6).

\subsection{Bifurcation}

For any family of smooth mappings a key role is provided by the bifurcation set of parameters for which the corresponding mapping exhibits a non-stable multigerm. Thus for the $p$-parameter family of trajectories $M_{\lambda}: L \times \mathbf{R}^{p} \rightarrow \mathbf{R}^{p}$ it comprises the set of tracing points $w \in \mathbf{R}^{p}$ for which the trajectory $M_{\lambda, w}$ exhibits a non-stable multigerm. The bifurcation set attached to the motion of a robotic device is fundamental, representing the boundary between qualitatively different types of mechanical behaviour. The kinematics literature does contain attempts to determine the (local and global) structure of bifurcation sets associated to mechanically simple examples, such as Müller's extensive analysis of the 4-bars in $[45,46]$. The basic approach has been to seek polynomial equations: however that runs into the general difficulty that the zero locus may be larger than the bifurcation set in question. One of the strengths of the singular approach is that the unfolding theory determines the local structure of the bifurcation set up to diffeomorphism (or in a few cases just homeomorphism). More precisely we have the next result, using the Basic Transversality Lemma and the standard result in singularity theory [40, page 190], that any versal unfolding of an $\mathcal{A}$-finite germ is equivalent to a suspension of a miniversal unfolding. Thus the local study of the bifurcation set of $M_{\lambda}$ reduces to the analysis of miniversal unfoldings for the relevant normal forms.

Theorem 3.1 Let $\lambda: N \rightarrow S E(p)$ be a generic Euclidean motion. Then the germ (at a finite set of points) of any trajectory is versally unfolded by $M_{\lambda}$, and the unfolding is equivalent to a suspension of a miniversal unfolding of the associated normal form. In particular, the local structure of the bifurcation set for $M_{\lambda}$ is determined by that for the miniversal unfolding.

Incidentally, the properties of trajectories described here are $\mathcal{I}$-invariant. That is, if $\lambda_{1}, \lambda_{2}$ are $\mathcal{I}$-equivalent via the invertible germ $\phi$ and elements $h, k \in S E(p)$, then the trajectory under $\lambda_{1}$ of $x \in \mathbf{R}^{p}$ is $\mathcal{A}$-equivalent to that of $k . x$ under $\lambda_{2}$. Moreover, if $M_{\lambda_{1}}$ versally unfolds the one trajectory then $M_{\lambda_{2}}$ does the other.

A major goal of the subject is to establish good algorithms leading to computer graphic renderings of bifurcation sets, a programme which has hardly begun. The idea of the instantaneous singular set discussed in Section 4 leads in principle to an algorithmic approach for some spatial bifurcation sets, but we are not aware of any resulting computer implementations. 


\section{First Order Invariants}

Before considering the singularities on trajectories it is worth detailing what information can be ascertained concerning the locus of tracing points possessing singular trajectories. One of the oldest intuitions of kinematics is that when a plane lamina moves with one dof there is at any instant a point, the instantaneous centre of rotation, whose trajectory is singular at that instant. Likewise, when a spatial body moves with one dof there is at any instant a line, the instantaneous axis of rotation. However, in the spatial case points on the axis do not have singular trajectories unless a certain $\mathcal{I}$-invariant (the pitch) is at that instant zero. The step from planar to spatial motions is not simply an increase of one in the dimension of the ambient space - there is a more fundamental difference, namely that in the classical list of simple Lie groups, the rotation subgroups of $S E(2)$ and $S E(3)$ lie in different series.

\subsection{Instantaneous Singular Sets}

For a 1 -manifold $N$, there are $\mathcal{I}$-invariant stratifications of the jet bundles $J^{k}(N, S E(p))$ with $k=1,2$ to which transversality of the jet extension of a motion implies the following [12]. For $p$ even, there is a unique instantaneous centre, except at a discrete set of points in the configuration space $N$ where the instantaneous rotational component vanishes; moreover the locus of instantaneous centres will be an immersion in $\mathbf{R}^{p}$. For $p$ odd, there is a unique instantaneous axis of rotation for each point in $N$ and the locus of those lines form a non-cylindrical ruled surface. Moreover there is a discrete set in $N$ at which the axis consists of singular points of trajectories. By virtue of the Basic Transversality Lemma these properties are generic. The generalization of these ideas to motions with several dof is hardly touched upon in the classical literature on kinematics. We introduce the following:

Definition 4.1 Given a motion germ $\lambda: N, x \rightarrow S E(p)$, e the instantaneous singular set (ISS) of $\lambda$ at $x$ is defined to be $I_{x}=\left\{q \in \mathbf{R}^{p} \mid M_{\lambda, q}\right.$ singular at $\left.x\right\}$.

For 1-dof planar (spatial) motions this yields the instantaneous centre of rotation (axis at pitch zero) alluded to above. A more explicit determination of the ISSs can be arrived at as follows. There is no loss in generality in assuming that $\lambda(x)=e$, the identity element of $S E(p)$. Now if $\alpha$ is the action of $S E(p)$ on $\mathbf{R}^{p}$ then for $q \in \mathbf{R}^{p}$, let $\alpha_{q}: S E(p) \rightarrow \mathbf{R}^{p}$ be the map $\alpha_{q}(g)=\alpha(g, q)=g \cdot q$. We may write the trajectory as a composite: $M_{\lambda, q}=\alpha_{q} \circ \lambda$. Assuming $\lambda$ itself to be non-singular and applying the Chain Rule and the Rank Theorem, the required condition for $q \in I_{x}$ is

$$
\operatorname{dim}\left(\operatorname{im} T_{x} \lambda \cap \operatorname{ker} T_{e} \alpha_{q}\right)>\max \{0, n-p\}
$$

The kernel of $T_{e} \alpha_{q}$ is the tangent space at $e$ to the subgroup of $S E(p)$ fixing $q$. In the planar case this condition is easy to interpret. Generically, for $n=2$ the ISS is an affine line, the locus of points $q$ for which the (2-dimensional) image of the tangent map of $\lambda$ has non-trivial intersection with the tangent space to the one-parameter subgroup of $S E(2)$ fixing $q$. For $n \geq 3$, singular trajectories are only possible when the motion itself has a singularity. In order to tackle the spatial case we shall need to explore more thoroughly the first-order $\mathcal{I}$-invariants of motion germs. 


\subsection{Screw Systems}

In the spatial case, the generalisation of the one dof instantaneous screw about an invariant axis to motions with several dof is known as a 'screw system'. The most significant early reference, virtually never quoted in the kinematics literature, is Klein's 1872 paper [38]. This work contains the germ of an idea central to understanding screw systems, namely the way in which they sit relative to the "Klein complex". The development of a general theory was undertaken in Ball's treatise [3], which concentrated almost exclusively on the least degenerate cases. The screw system concept was revived in the engineering community by Hunt [35], who pointed out that the mechanically interesting cases were virtually always special in some sense, and proposed a classification. However, the equivalence relation underlying the listing was not explicit, raising doubts about its completeness. This situation was rectified in [27], and a classification was described intrinsically by the present authors in [14] following the original philosophy of Klein. In [15] we showed that the listing gave rise to natural Whitney stratifications, and gave complete descriptions of the specialisations. These results are outlined below.

It is no restriction in the definition of $\mathcal{I}$-equivalence to suppose that $g_{1}=g_{2}=e$, in which case $\mathcal{I}$-equivalence requires $k=h^{-1}$. Let $\lambda: N, x \rightarrow S E(3), e$ be a motion germ. Write $s e(3)$ for the Lie algebra of the Euclidean group $S E(3)$. Changes of coordinates at the source $x \in N$ leave the image of the differential $T_{x} \lambda: T_{x} N \rightarrow \operatorname{se}(3)$ invariant, while the derivative at the target $e \in S E(3)$ of conjugation gives rise to the adjoint action of the group on its Lie algebra. Since this action is linear it induces an action on subspaces of $s e(3)$ of given dimension $k$ : thus if two motion germs are $\mathcal{I}$-equivalent the images of their derivatives lie in the same orbit under this action. The objects of interest are the vector subspaces of $s e(3)$, representing images of derivatives of motions. A $k$-dimensional subspace $(1 \leq k \leq 6)$ of $s e(3)$ is called a motor system or $k$-system, and can be viewed as an element of the Grassmannian $G(k, s e(3))$ of $k$-dimensional subspaces. Alternatively, we may think of a $k$-system as a $(k-1)$-dimensional projective subspace of the projective Lie algebra Pse(3), the screw space: in that case, projective subspaces are screw systems.

We need to express the action more explicitly. $S E(3)$ is isomorphic to a semi-direct product $S O(3) \times{ }_{s} T(3)$, with $T(3)$ the translation subgroup, so we can think of an element of $S E(3)$ as a pair $(A, a)$ with $A \in S O(3)$ and $a \in T(3)$; correspondingly the Lie algebra $s e(3)$ is isomorphic to a semi-direct product $s o(3) \times{ }_{s} t(3)$ of Lie algebras, where we can identify $t(3)$ with $\mathbf{R}^{3}$. Thus elements of $s e(3)$ can be represented either by a pair $(B, b)$ where $B$ is a $3 \times 3$ skew-symmetric matrix and $b \in \mathbf{R}^{3}$, or by a pair of 3 -vectors $(u, v)$, where $u$ is the unique vector for which $B x=u \wedge x(u=0$ when $B=0)$, and $v=b$. The latter are known as motor coordinates for se(3), or screw coordinates for $P s e(3)$. In these coordinates the adjoint action is given by

$$
(A, a) \cdot(u, v)=(A u, A v-A u \wedge a) .
$$

Two basic invariants have long been known in the subject, namely the Killing form $\langle u, u\rangle$, and the Klein form $\langle u, v\rangle$, where $\langle$,$\rangle represents the standard scalar product on \mathbf{R}^{3}$. The group $S E(p)$ behaves like a geometrically reductive group, in that the ring of invariant polynomials for the adjoint action is finitely generated. In [14] we gave explicit generators, the form depending on the parity of $p$. When $p=3$ the ring is generated by the Klein and Killing forms, a result implicit in the subject but for which we are not aware of any prior reference.

Since the Killing and Klein forms are both of degree 2, their ratio $h=\langle u, v\rangle /\langle u, u\rangle$ is an invariant of the action induced by the adjoint action on the screw space, called the pitch. When $u=0$ we define $h=\infty$. The concept of "pitch" is directly related to its usual physical meaning as follows. Each element of the Lie algebra defines a Killing vector field on $\mathbf{R}^{3}$ whose orbits are setwise invariant 
under projectivisation in $s e(3)$. In general $(h \neq 0, \infty)$, the orbits are helices about an invariant axis (the instantaneous screw axis) with common pitch $h$. When $h=0$ the helices degenerate to circles about a fixed axis, corresponding to infinitesimal rotation, and when $h=\infty$ the helices degenerate to lines parallel to the axis, corresponding to instantaneous translation. There is an obvious connection with the $\mathrm{R}-, \mathrm{H}-$ and $\mathrm{P}-$-joints of the introduction.

It is natural and profitable to foliate the screw space by pitch. Formally, define the pitch quadric

$$
Q_{h}(u ; v)=\langle u, v\rangle-h\langle u, u\rangle
$$

whose zero set is the union of the screws of pitch $h$, and those of pitch $\infty$. Note that $Q_{\infty}$ is degenerate: it is a projective 2-plane (the vertex of the complex quadric $Q_{\infty}$ ) contained in all the $Q_{h}$. A special role is played by the quadric $Q_{0}$ containing all screws of pitch zero. Mathematically, $Q_{0}$ is the Klein quadric in real projective 5-space, whose points represent lines in projective $3-$ space, where now $(u ; v)$ correspond to Plücker line coordinates. One simply identifies a screw of pitch zero with its fixed axis. Under this identification, subsets of $Q_{0}$ correspond to ruled subsets of $\mathbf{P} \mathbf{R}^{3}$ as in Table 2. In particular the Klein quadric has a double ruling by families of 2-planes, namely the classical $\alpha$ - and $\beta$-planes. The importance of $Q_{0}$ in engineering terms is that revolute joints are more widely used than others.

\begin{tabular}{|c|c|}
\hline$Q_{0}$ & PR $^{3}$ \\
\hline \hline point & line \\
$\alpha$-plane & bundle of lines through a point \\
$\beta$-plane & bundle of lines in a plane \\
line & planar pencil of lines \\
conic & ruled hyperboloid \\
\hline
\end{tabular}

Table 2: Correspondence between $Q_{0}$ and $\mathbf{P R}^{3}$.

The quadrics $Q_{h}$ form a pencil and thus a quadratic complex, known as the Klein complex. It is somewhat degenerate, since it contains just one singular member, namely $Q_{\infty}$. The complex is central to an understanding of screw systems. The key intuition is that any screw system will intersect the Klein complex in a pencil of quadrics of lower dimension, whose type provides an invariant. Thus a 2-system will intersect the complex in a pencil of points, a 3 -system in a pencil of conics, and so on. The details of the classification appear in [14,15]. The most interesting case is the (self-dual) 3-systems. The most general type is labelled $\mathrm{IA}_{1}$, for which a projective basis can be put in the normal form

$$
\left(1,0,0 ; h_{\alpha}, 0,0\right) \quad\left(0,1,0 ; 0, h_{\beta}, 0\right) \quad\left(0,0,1 ; 0,0, h_{\gamma}\right) .
$$

The moduli $h_{\alpha}, h_{\beta}, h_{\gamma}$ are the principal pitches: namely those for which the 3 -system and the pitch quadric intersect in a reducible conic; we may assume them to be ordered, say $h_{\alpha}>h_{\beta}>$ $h_{\gamma}$. A refinement of the classification emphasises the role of screws of pitch zero. Technically this is achieved by weakening the adjoint action, allowing Euclidean similarities in place of rigid displacements. This enables us to reduce the number of pitch moduli by one. The subtypes of the $\mathrm{IA}_{1}$ type (in an obvious notation) are as follows:

$$
\mathrm{IA}_{1}^{+++}, \mathrm{IA}_{1}^{++0}, \mathrm{IA}_{1}^{++-}, \mathrm{IA}_{1}^{+0-}, \mathrm{IA}_{1}^{+--}, \mathrm{IA}_{1}^{0--}, \mathrm{IA}_{1}^{---} .
$$

Let us now return to the determination of instantaneous singular sets and concentrate on this case $p=3$. They can be derived from the condition (2) and the refined classification of 3-systems. The 
image of the derivative of the motion is, projectively, just the associated screw system while the kernel of $T_{e} \alpha_{q}$ is projectively the $\alpha$-plane $A_{q}$ in $Q_{0}$ consisting of the bundle of lines through $q$. Therefore the ISS is the set of $q$ for which the screw system intersects $A_{q}$ in a projective subspace of dimension $\geq \max \{0, n-3\}$. Moreover, this number (plus one) gives the corank of the singularity on the trajectory of $q$. The ISS associated to a given screw system type can now be determined via a straightforward computation based on the normal form. The details will appear in [16]. For example, in the most general case of a 3-dof spatial motion $\lambda: N, x \rightarrow S E(3), e$ the screw system is of type $\mathrm{IA}_{1}$ with no principal pitch zero; its intersection with $Q_{0}$ is an irreducible conic (or empty) and hence (Table 2) the ISS is a hyperboloid in $\mathbf{R}^{3}$.

\subsection{Regularity and Genericity}

The listings are based on non-canonical choices of strata, raising the question of naturality. Explicitly, do we obtain Whitney regular stratifications of the Grassmannians $G(k, s e(3))$ ? Given that the classes are unions of orbits of a Lie group acting on the Grassmannian, all the necessary information is obtained by constructing minimal transversals (unfoldings) to orbits at normal forms. Finding transversals is a lengthy but essentially mechanical process whose details can be found in [15]. By inspection one finds that each stratum intersects a transversal in an affine subspace, so is smooth. Its codimension is the minimal number of unfolding parameters less the number of moduli. Further analysis of the transversals establishes specialisations between types, and standard arguments on regularity show that in each dimension the stratification (and its refinement) is Whitney regular.

These results about stratifications yield a genericity theorem relating to screw systems for spatial motions. An $n$-dof motion $\lambda: N \rightarrow S E(3)$ gives rise to a jet extension $j^{1} \lambda: N \rightarrow J^{1}(N, S E(3))$. There is no reason to suppose a priori that $\lambda$ is regular, however in the case $n \leq 3$, on an open and dense set in $C^{\infty}(N, S E(3))$ motions are immersive. In any case, we may partition $J^{1}(N, S E(3))$ into the sets $\Sigma^{k}$ consisting of jets of rank $k$ with $0 \leq k \leq n$. A stratification of $G(k, s e(3))$ induces a stratification $\mathcal{S}_{k}$ of $\Sigma^{k}$ via (left) translation in the group, and hence a stratification $\mathcal{S}$ of the jet bundle. $\lambda$ is said to be 1 -generic when it is transverse to $\mathcal{S}$. The Thom Transversality Theorem gives:

Theorem 4.1 The 1-generic motions $\lambda: N \rightarrow S E(3)$ form a residual set in $C^{\infty}(N, S E(3))$, endowed with the Whitney $C^{\infty}$ topology.

Indeed, the regularity of the stratifications ensures that for $n \leq 3$ the 1 -generic motions form an open and dense set. A 1-generic motion will exhibit only strata of codimension $\leq n$, which restricts considerably the types of screw systems one expects to encounter. Explicit lists of these screw systems can be found in [14].

\section{$5 \quad$ Trajectories of Planar Motions}

Descriptions of generic $n$-dof planar motions, including complete listings of the (mono and multi) germs which can appear on their trajectories, fall into three distinct cases, $n=1$ treated in [23], $n=2$ treated in [24] and $n \geq 3$ treated in [17]. Planar motions are maps into the 3 -dimensional group $S E(2)$. For $n=1$ a generic planar motion is an embedding; for $n=2$ it may exhibit crosscaps, as well as singular multigerms; and for $n \geq 3$ it may exhibit folds, cusps and swallowtails. 
We give an account of the trajectory singularities that can occur generically and their connections with the $\mathcal{A}$-type and ISS of the motion. Much of the listing appears in the existing literature and we cite sources as we proceed.

\subsection{One dof Planar Motions}

The case $n=1$ is significant since it represents a formalization of a set of intuitions familiar to any working kinematician. An account for an engineering audience appeared in [21]. One simplifying feature is that general 1 -dof motions do not themselves exhibit singularities.

Theorem 5.1 ([23]) For a residual set of 1-dof motions $\lambda: N \rightarrow S E(2)$ of the plane, any multigerm of a trajectory is $\mathcal{A}$-equivalent to one of the normal forms in Table 3 below.

\begin{tabular}{|l|l|l|c|l|}
\hline type & description & normal form & cod & versal unfolding \\
\hline \hline$A_{0}$ & smooth point & $(s, 0)$ & 0 & \\
$A_{2}$ & ordinary cusp & $\left(s^{2}, s^{3}\right)$ & 1 & $\left(s^{2}, s^{3}+a s\right)$ \\
$A_{4}$ & ramphoid cusp & $\left(s^{2}, s^{5}\right)$ & 2 & $\left(s^{2}, s^{5}+a s^{3}+b s\right)$ \\
\hline$A_{1}$ & node & $(s, 0 ; 0, t)$ & 0 & \\
$A_{3}$ & tacnode & $\left(s, 0 ; t, t^{2}\right)$ & 1 & $\left(s, a ; t, t^{2}\right)$ \\
$A_{5}$ & flecnode & $\left(s, 0 ; t, t^{3}\right)$ & 2 & $\left(s, a ; t, t^{3}+b t\right)$ \\
$D_{5}$ & cusp plus line & $\left(s, 0 ; t^{3}, t^{2}\right)$ & 2 & $\left(s, a ; t^{3}+b t, t^{2}\right)$ \\
\hline$D_{4}$ & triple point & $(s, 0 ; 0, t ; u, u)$ & 1 & $(s, 0 ; 0, t ; u, u+a)$ \\
$D_{6}$ & tacnode plus line & $\left(s, 0 ; 0, t ; u, u^{2}\right)$ & 2 & $\left(s, a s+b ; 0, t ; u, u^{2}\right)$ \\
\hline$\tilde{E}_{7}$ & quadruple point & $(t, 0 ; 0, s ; u, u ; v, \lambda v)$ & 3 & $(t, 0 ; 0, s ; u+a, u ; v+b, \lambda v)$ \\
\hline
\end{tabular}

Table 3: Local models for 1-dof planar motions.

The listing of the monogerms was first obtained as part of the listing of $\mathcal{A}$-simple monogerms of plane parametrized curves in the unpublished paper [7], which appeared later as [9]. The symbols $A_{0}, A_{1}, \ldots$ in the first column of Table 3 are the $\mathcal{K}$-types of germs at the origin of smooth functions on the plane whose zero-set is the image of the multigerm: it was an observation of Bruce [9] that for (irreducible) monogerms of parametrized plane curves there is a one-to-one correspondence between $\mathcal{A}$-equivalence classes of monogerms and $\mathcal{K}$-equivalence classes of defining equations for the image. All but one of the local models are smoothly equivalent to the unfoldings of the trajectory germs obtained by varying the position of the tracing point. The only exception is the $\mathcal{A}$-unimodular family $\tilde{E}_{7}$ for which we only have topological equivalence. (It is assumed that the modulus $\lambda \neq 0,1$.) As to multigerms, the case when both branches are immersive appeared in [57]. [23] provides a convenient reference for pictures of the versal unfoldings. In particular, the ramphoid cusp illustrates the danger (mentioned in Section 3.4) of trying to obtain local pictures of bifurcation sets by finding defining equations. In the unfolding $(a, b)$-plane the line $b=0$ (with the origin deleted) represents cusps, whilst the half parabola $4 b=a^{2}, a<0$ represents tacnodes. However an algebraic curve containing these curves in its zero locus has to have equation $b\left(4 b-a^{2}\right)=0$, exhibiting two smooth branches tangent at the origin. Beyond the listing in Table 3 lie germs of higher codimension. The first such families are those corresponding to the $\mathcal{K}$-type of the germ $t, 0 \mapsto t^{3}, 0$; the $\mathcal{A}$-types are labelled $E_{6 k}, E_{6 k+2}$ in [9]. It was shown in [12] that these types can 
only occur when the centrode curve (the locus of ISSs) itself has a singularity. This presages further relations between singularity type and the ISSs for 2-dof motions.

In principle there are three bifurcation curves associated to a 1-dof motion, corresponding to the three multigerms of codimension 1, namely the cusp, tacnode and triple point curves. For the planar 4-bar, the cusp curve, not explicitly available from the analysis in Section 2, is nevertheless easy to render because it is traced by the instantaneous centre of rotation, the point of intersection of the first and third links. A robust algorithm for the tacnode curve results from an understanding [28] of the geometry in Section 2. And the triple point curve is empty, except when the Grashof relations are satisfied, when it can be determined explicitly. At isolated points on these bifurcation curves one expects to encounter codimension 2 singularities. A detailed analysis [11] of the geometry rules out all of these for the 4-bar except the ramphoid cusp and flecnode.

\subsection{Two dof Planar Motions}

The study of 2-dof planar motions already lies significantly beyond the confines of classical kinematics. The trajectories are 2-dimensional subsets of the plane. In particular, they exhibit singularities at their boundaries - from the roboticist's point of view these are the degeneracies which can appear on "workspace boundaries". An account of the results for an engineering audience appears in [22].

Theorem 5.2 ([24]) For a residual set of 2-dof motions $\lambda: N \rightarrow S E(2)$ of the plane, any multigerm of a trajectory is $\mathcal{A}$-equivalent to one of the normal forms in Table 4.

\begin{tabular}{|l|l|c|c|}
\hline name & normal form & cod & versal unfolding \\
\hline \hline immersion & $(x, y)$ & 0 & \\
fold & $\left(x, y^{2}\right)$ & 1 & \\
cusp & $\left(x, x y+y^{3}\right)$ & 1 & \\
\hline lips & $\left(x, y^{3}+x^{2} y\right)$ & 1 & $\left(x, y^{3}+x^{2} y+a y\right)$ \\
beaks & $\left(x, y^{3}-x^{2} y\right)$ & 1 & $\left(x, y^{3}-x^{2} y+a y\right)$ \\
swallowtail & $\left(x, x y+y^{4}\right)$ & 1 & $\left(x, x y+y^{4}+a y^{2}\right)$ \\
\hline goose & $\left(x, y^{3}+x^{3} y\right)$ & 2 & $\left(x, y^{3}+x^{3} y+a y+b x y\right)$ \\
butterflies & $\left(x, x y+y^{5} \pm y^{7}\right)$ & 2 & $\left(x, x y+y^{5} \pm y^{7}+a y^{2}+b y^{3}\right)$ \\
gulls & $\left(x, x y^{2}+y^{4}+y^{5}\right)$ & 2 & $\left(x, x y^{2}+y^{4}+y^{5}+a y+b y^{3}\right)$ \\
\hline sharksfin & $\left(x^{2}+y^{3}, y^{2}+x^{3}\right)$ & 2 & $\left(x^{2}+y^{3}+a y, y^{2}+x^{3}+b x\right)$ \\
deltoid & $\left(x^{2}-y^{2}+x^{3}, x y\right)$ & 2 & $\left(x^{2}-y^{2}+x^{3}+a x, x y+b x\right)$ \\
\hline
\end{tabular}

Table 4: Monogerms for 2-dof planar motions.

The stable monogerms of the plane (the immersion, fold and cusp) were listed in the seminal paper of Whitney [56]. The corank 1 germs were listed independently by Arnold [2], du Plessis [49] and Gaffney [20]. The corank 2 germs do appear in the existent literature [49, page 134], but require some work to tie them into the above listing. The sobriquets for the corank 2 normal forms reflect the forms of their versal unfoldings: for the sharksfin these are essentially sections of the hyperbolic umbilic, and for the deltoid sections of the elliptic umbilic. 
Can all the germs in Table 4 appear as trajectory germs associated to 2-dof planar motions? A generic 2-dof planar motion is an immersed surface in $S E(2)$, except for possibly a discrete set of cross-cap points. The first observation to make is that at an immersive point of the motion only corank 1 germs can appear on the trajectories: moreover, putting the motion germ into a normal form, we see that any corank 1 germ of the plane, generic or not, can appear as a trajectory germ. What happens at a cross-cap point? The following answer appeared independently in [55] in a rather different context.

Theorem 5.3 ([29]) In the cross-cap case the only generic singularity types which can appear on trajectories are folds, cusps, swallowtails, butterflies, sharksfin and deltoid.

That all these types can appear is established by putting the cross-cap into its standard normal form $\left(s, s t, t^{2}\right)$ and then, for each generic type, constructing an explicit submersive germ $\mathbf{R}^{3}, 0 \rightarrow \mathbf{R}^{2}, 0$ whose composite with the standard cross-cap is of the given type. That the remaining types (the lips, beaks, goose and gulls) cannot appear is established similarly: one takes the composite of an arbitrary submersive germ with the standard cross-cap, and follows the classification of planar germs.

For 2-dof planar motions there are three bifurcation curves associated to monogerm types, namely the lips, beaks and swallowtail. For the lips and beaks types the bifurcation curves are described as envelopes of 2-parameter families of lines in the following result [29].

Theorem 5.4 Let $\lambda: N \rightarrow S E(2)$ be an immersive, generic motion. The envelope of the ISS lines of points in $N$ is the union of the closures of the lips and beaks bifurcation curves.

The key property isolating the lips and beaks types is that they are the only codimension 1 generic singularity types with non-smooth critical sets. This result opens up the possibility of rendering the lips and beaks bifurcation curves using computer algebra programs to determine an equation for the envelope. We are not aware of any similar approach for the swallowtail.

The complete listing of generic multigerm types [24] is given in Table 5. There are five bifurcation curves, corresponding to the three multigerm types of codimension 1 , namely the tacnode folds, the cusp plus fold, and the triple fold. Note that the tacnode folds are topologically distinct. A topological invariant is provided by the topological type of the set $T$ of points in the target for which each branch has exactly two pre-images: $T$ is connected for the ++ tacnode fold, disconnected for the +- tacnode fold, and empty for the -- tacnode fold.

The double 4-bar [29] in which one 4-bar is mounted on the coupler link of a second, provides a mechanically generated 2-dof motion which exhibits most of the generic singularity types in Table 4. Computer renderings of typical critical images, illustrating surprisingly complex behaviour, are reproduced in $[58,29]$. The double 4 -bar is an example of a composite planar motion, in the sense that two 1-dof motions (one for each 4-bar) are composed in $S E(2)$. This is typical of motions arising from serial devices such as robot arms. One would expect the behaviour of composite motions to be significantly simpler than those of general motions. Current work [29] indicates that they exhibit a limited range of generic trajectory types: for instance the deltoid singularity cannot appear for composite 2-dof planar motions. Corank 2 singularities admit a particularly neat description for composite planar motions, namely that the (moving) instantaneous centre for the first motion should coincide with the (fixed) instantaneous centre for the second motion, a condition with a very simple mechanical interpretation [29] for the double 4-bar. 


\begin{tabular}{|l|l|c|}
\hline description & normal form & cod \\
\hline \hline node fold & $\left(x, y^{2} ; X^{2}, Y\right)$ & 0 \\
\hline++ tacnode fold & $\left(x, y^{2} ; X, X^{2}+Y^{2}\right)$ & 1 \\
+- tacnode fold & $\left(x, y^{2} ; X, X^{2}-Y^{2}\right)$ & 1 \\
-- tacnode fold & $\left(x, y^{2} ; X,-X^{2}-Y^{2}\right)$ & 1 \\
cusp plus fold & $\left(x, y^{2} ; X Y+X^{3}, Y\right)$ & 1 \\
\hline cusp plus tangent & $\left(x, y^{2} ; X, X Y+Y^{3}\right)$ & 2 \\
flecnode fold & $\left(x, y^{2} ; X, Y^{2}+X^{3}\right)$ & 2 \\
lips plus fold & $\left(x, y^{2} ; X^{3}+X Y^{2}, Y\right)$ & 2 \\
beaks plus fold & $\left(x, y^{2} ; X^{3}-X Y^{2}, Y\right)$ & 2 \\
swallowtail plus fold & $\left(x, y^{2} ; X Y+X^{4}, Y\right)$ & 2 \\
cusp plus cusp & $\left(x, x y+y^{3} ; X Y+X^{3}, Y\right)$ & 2 \\
\hline \hline triple fold & $\left(x, y^{2} ; X^{2}, Y ; \tilde{x}, \tilde{x}+\tilde{y}^{2}\right)$ & 1 \\
\hline node fold plus cusp & $\left(x, y^{2} ; X^{2}, Y ; \tilde{x}, \tilde{x}+\tilde{x}+\tilde{y} \tilde{y}^{3}\right)$ & 2 \\
++ tacnode fold plus fold & $\left(x, y^{2} ; X^{2}, Y ; \tilde{x}, \tilde{x}^{2}+\tilde{y}^{2}\right)$ & 2 \\
+- tacnode fold plus fold & $\left(x, y^{2} ; X^{2}, Y ; \tilde{x}, \tilde{x}^{2}-\tilde{y}^{2}\right)$ & 2 \\
-- tacnode fold plus fold & $\left(x, y^{2} ; X^{2}, Y ; \tilde{x},-\tilde{x}^{2}-\tilde{y}^{2}\right)$ & 2 \\
\hline \hline quadruple fold & $\left(x, y^{2} ; X^{2}, Y ; \tilde{x}, \tilde{x}+\tilde{y}^{2} ; \tilde{X}, \lambda \tilde{X} \pm \tilde{Y}^{2}\right)$ & 3 \\
\hline
\end{tabular}

Table 5: Multigerms for 2-dof planar motions.

\subsection{Planar Motions with $\geq 3$ dof}

The results of Section 5.2 can be extended [17] to motions with many dof via a basic Splitting Lemma [50] analogous to Thom's better known result for functions [53, pages 59-60]. Like Thom's result it is easily established by an inductive argument on the $k$-jet (starting at $k=2$ ) using the Complete Transversal Lemma of [10] for the induction step.

Theorem 5.5 (Splitting Lemma) Let $F: \mathbf{R}^{n}, 0 \rightarrow \mathbf{R}^{2}, 0$ be an $\mathcal{A}$-finite germ of corank 1 . Then $F$ is $\mathcal{A}$-equivalent to a germ $\left(x, f\left(x, y_{1}, \ldots, y_{r}\right) \pm t_{1}^{2} \pm \ldots \pm t_{s}^{2}\right)$ where $f\left(x, y_{1}, \ldots, y_{r}\right)$ has zero 1 -jet and $f\left(0, y_{1}, \ldots, y_{r}\right)$ has zero 2 -jet.

In this situation we say that $F$ is stably $\mathcal{A}$-equivalent to the germ $\left(x, f\left(x, y_{1}, \ldots, y_{r}\right)\right)$. Under the hypotheses of the lemma the kernel of the differential of $F$ has dimension $(n-1)$, and the cokernel has dimension 1 , so the second intrinsic derivative [41, page 213] is represented by a single quadratic form, whose rank is the integer $s$. Thus $r$ and $s$ are $\mathcal{A}$-invariants of $F$. For $n \geq 3$ only corank 1 singularities appear generically: essentially one obtains the same list of corank 1 germs as in the 2-dof case, plus two $\mathcal{A}$-unimodular families. More precisely:

Theorem 5.6 For a residual set of $n$-dof planar motions $\lambda: N \rightarrow S E(2)$ with $n \geq 3$, any trajectory monogerm is stably $\mathcal{A}$-equivalent either to one of the corank 1 normal forms in Table 4 , or to one of the $\mathcal{A}$-unimodular families $\left(x, x y+y^{3}+b y^{2} z+z^{3} \pm y^{5}\right)$ where the modulus $b$ is distinct from two exceptional values $b=0, b=b_{0}$ with $4 b_{0}^{3}+27=0$. 
The $\mathcal{A}$-unimodular families (one for the + sign, and one for the - sign) defined by the displayed formula are dubbed the ephemera. For a fixed value of $b$ the germs can be distinguished by the determinant of their discriminant matrices [19]. They are the first $\mathcal{A}$-unimodular families in these dimensions, in the sense that there are only finitely many $\mathcal{A}$-types of strictly lower codimension specializing to them. The ephemera are best understood as 1-parameter unfoldings (with $x$ as the unfolding parameter) of the umbilic $y^{3}+b y^{2} z+z^{3} \pm y^{5}$. (For $b>b_{0}$ this is a $D_{4}^{-}$, and for $b<b_{0}$ a $D_{4}^{+}$, in the Arnold notation.) The full bifurcation sets for the umbilics are well-understood [54] and enable the specializations to the ephemera to be determined. The fold, cusp, lips, beaks and swallowtail all specialize to the ephemera: interestingly, the double and triple folds are the only multigerm specializations. It is a difficult exercise in computer graphics to obtain renderings of versal unfoldings for the ephemera. Some pictures can be found in [17]. Incidentally, one of them bears a striking resemblance to illustrations in biology texts of Ephemera danica, the common may-fly: hence the nomenclature.

Having established for $n \geq 3$ a list of $\mathcal{A}$-types $\mathbf{R}^{n}, 0 \rightarrow \mathbf{R}^{2}, 0$ of codimension $\leq 2$ we ask whether these germs can actually appear as germs of trajectories associated to $n$-dof planar motions. In contradistinction to the case $n<3$, a singular point on a trajectory can only arise from a singular point of the motion. Moreover the nature of the trajectory singularity will depend on the nature of the motion singularity. For a general $n$-dof planar motion $\lambda: N \rightarrow S E(2)$ with $n \geq 3$ the motion germ is stable, and by results of Morin [44], normal forms up to $\mathcal{A}$-equivalence are given by the 2 parameter unfoldings of the $A_{1^{-}}, A_{2}$-and $A_{3}$-singularities in Table 6 , where $q(t)= \pm t_{1}^{2} \pm \ldots \pm t_{n-3}^{2}$ for $n \geq 4$.

\begin{tabular}{|l|l|c|}
\hline name & normal form for $\lambda$ & cod \\
\hline \hline fold & $\left(x, y, z^{2}+q(t)\right)$ & 1 \\
cusp & $\left(x, y, z^{3}+y z+q(t)\right)$ & 2 \\
swallowtail & $\left(x, y, z^{4}+y z+x z^{2}+q(t)\right)$ & 3 \\
\hline
\end{tabular}

Table 6: Stable germs of planar motions.

The following results from [17] yield a complete answer to the above question. In particular, they show that the ephemera types can only appear at singular germs of the motion of cusp or swallowtail type.

Theorem 5.7 At a singular motion germ of (a) fold type, (b) cusp type, (c) swallowtail type only the following trajectory germs can appear: (a) $\mathcal{A}$-simple types, i.e. those stably $\mathcal{A}$-equivalent to the corank 1 germs in Table 4; (b) fold, cusp and ephemera types; (c) fold, swallowtail and ephemera types. Moreover each such trajectory type can be exhibited for some singular motion germ of the given type.

\section{The Spatial Case}

The study of $n$-dof spatial motions is a core concern of engineering robotics. From the mathematical point of view it is more challenging than the planar case, and in some measure more interesting, in that the study of the motion itself (the object of Section 4) begins to play a substantial role 
in one's understanding. There is as yet no complete listing of trajectory singularities, but we will describe briefly what is known.

\subsection{One dof Spatial Motions}

The case $n=1$ was covered in [23] and gives rise to the remarkably short list in Table 7: the two monogerms represent the beginning of the list of $\mathcal{A}$-simple monogerms of space curves obtained in $[25]$.

\begin{tabular}{|l|l|l|c|l|}
\hline type & description & normal form & cod & versal unfolding \\
\hline \hline$A_{0}$ & smooth point & $(s, 0,0)$ & 0 & \\
$A_{2}$ & simple cusp & $\left(s^{2}, s^{3}, 0\right)$ & 2 & $\left(s^{2}, s^{3}+a s, b s\right)$ \\
\hline \hline$A_{1}$ & node & $(s, 0,0 ; t, t, 0)$ & 1 & $(s, 0,0 ; t, t, a)$ \\
$A_{3}$ & tacnode & $\left(s, 0,0 ; t, t^{2}, 0\right)$ & 3 & $\left(s, a, b+c s ; t, t^{2}, 0\right)$ \\
\hline \hline$L_{3}^{3}$ & triple point & $(s, 0,0 ; 0, t, 0 ; 0,0, u)$ & 3 & $(s, a, 0 ; 0, t, b ; c, 0, u)$ \\
\hline
\end{tabular}

Table 7: Multigerms for 1-dof spatial motions.

Bifurcation takes place on a nodal surface on which lies a cuspidal curve, and exceptional points distinct from the curve corresponding to tacnodes and triple points: however as we have seen in Section 4 the cuspidal curve is forced by the geometry of the motion to take a special form, namely to be a union of lines. The versal unfoldings of these types have immediate geometric content. Perhaps the most interesting case is that of the tacnode, where the bifurcation set is the Whitney umbrella in the unfolding $(a, b, c)$-space defined by $a c^{2}=b^{2}$, with "handle" the $a$-axis. All the unfoldings represent a parabola and a line. Off the umbrella the line and the parabola fail to meet. On the umbrella (but off the handle) they meet in a single point. On the handle the line and parabola are coplanar: for $a>0$ they meet in two distinct points, and for $a<0$ they fail to meet.

\subsection{Two dof Spatial Motions}

The listing of trajectory monogerms for 2-dof spatial motions in Table 8 is subsumed in Mond's listing [42] of singularities of generic smooth mappings from a surface into a 3-manifold. All the germs are $\mathcal{A}$-simple, with the exception of the $\mathcal{A}$-unimodular family $P_{3}(c)$ with modulus $c$ having four exceptional values $c=0, c=\frac{1}{2}, c=1$ or $c=\frac{3}{2}$. The only generic singularity type is the cross-cap type $S_{0}$. Cross-caps have certainly been observed in the engineering literature as stable phenomena appearing on coupler surfaces of the RSSR linkage [51].

The only generic multigerms are double points, which appear on curves in the plane, and triple points, which appear as discrete points on those curves. The number $C$ of cross-caps, and the number $T$ of triple points, appearing in a generic versal unfolding are basic invariants associated to any of the germs in Table 8. The geometry of the germ is largely determined by the double point curve in the source. For germs of the form $\left(x, y^{2}, y p\left(x, y^{2}\right)\right)$ this curve has equation $p\left(x, y^{2}\right)=0$ so is particularly easy to study. Following initial work in [42], the bifurcations of the germs in Table 8 were studied in [26], giving rise to a series of computer generated renderings. For instance 


\begin{tabular}{|l|l|c|}
\hline type & normal form & cod \\
\hline \hline$S_{0}$ & $\left(x, y^{2}, x y\right)$ & 0 \\
$S_{1}^{+}$ & $\left(x, y^{2}, y^{3}+x^{2} y\right)$ & 1 \\
$S_{1}^{-}$ & $\left(x, y^{2}, y^{3}-x^{2} y\right)$ & 1 \\
$S_{2}$ & $\left(x, y^{2}, y^{3}+x^{3} y\right)$ & 2 \\
$S_{3}^{+}$ & $\left(x, y^{2}, y^{3}+x^{4} y\right)$ & 3 \\
$S_{3}^{-}$ & $\left(x, y^{2}, y^{3}-x^{4} y\right)$ & 3 \\
\hline$B_{2}^{+}$ & $\left(x, y^{2}, x^{2} y+y^{5}\right)$ & 2 \\
$B_{2}^{-}$ & $\left(x, y^{2}, x^{2} y-y^{5}\right)$ & 2 \\
$B_{3}^{+}$ & $\left(x, y^{2}, x^{2} y+y^{7}\right)$ & 3 \\
$B_{3}^{-}$ & $\left(x, y^{2}, x^{2} y-y^{7}\right)$ & 3 \\
\hline$C_{3}^{+}$ & $\left(x, y^{2}, x y^{3}+x^{3} y\right)$ & 3 \\
$C_{3}^{-}$ & $\left(x, y^{2}, x y^{3}-x^{3} y\right)$ & 3 \\
\hline$H_{2}$ & $\left(x, y^{3}, x y+y^{5}\right)$ & 2 \\
$H_{3}$ & $\left(x, y^{3}, x y+y^{8}\right)$ & 3 \\
\hline$P_{3}(c)$ & $\left(x, x y+y^{3}, x y^{2}+c y^{4}\right)$ & 3 \\
\hline
\end{tabular}

Table 8: Monogerms for 2-dof spatial motions.

the germs $P_{3}(c)$ have versal unfoldings

$$
\left(x, x y+y^{3}+a y, x y^{2}+c y^{4}+b y+d y^{3}\right)
$$

and a starting point is to determine the subsets of $(a, b, c, d)$-space which give rise to cross-caps and triple points. For a fixed value of the modulus $c$ we obtain (for cross-caps) a cubic surface in $(a, b, d)$-space, and (for triple points) a quartic surface. The weighted homogeneity of the germ $P_{3}(c)$ reduces the problem of understanding the intersection of these two surfaces in the plane section $d=1$. The sections are cuspidal cubic curves, whose intersections depend (interestingly) not just on the four exceptional values of $c$ listed above, but in addition two further values $c=\frac{4}{3}$ and $c=2$. The way in which these cubics intersect is illustrated in [26, Figure 16].

The listing process for multigerms of higher codimension was started in [42], continued in [33], and was recently extended in [34]: for multigerms with several branches the number of moduli increases rather quickly.

\subsection{Spatial Motions with $\geq 3$ dof}

Results here are relatively limited. The best understood case is $n=3$. The stable monogerms in these dimensions are classical, and given by the first four entries in Table 9 . The first listing beyond this appears in Arnold's work on the evolution of galaxies, where the germs were gradients of smooth functions in three variables. Bruce [8] considered arbitrary germs, and obtained a complete list of monogerms up to codimension 1 given by the remaining entries in Table 9 . All these germs are $\mathcal{A}$-simple, and of corank 1, so appear in the recent classification of Marar and Tari [39].

Beyond this point the listing becomes a difficult technical exercise. A complete list up to codimension 3 was given by Hawes [32] using the computer algebra program TRANSVERSAL developed [37] in Liverpool by Kirk. The resulting list represents over fifty types, and includes a number of 


\begin{tabular}{|l|c|}
\hline normal form & cod \\
\hline$(x, y, z)$ & 0 \\
$\left(x, y, z^{2}\right)$ & 0 \\
$\left(x, y, y z+z^{3}\right)$ & 0 \\
$\left(x, y, y z+x z^{2}+z^{4}\right)$ & 0 \\
\hline$\left(x, y z, y^{2}+z^{2}+x y\right)$ & 1 \\
$\left(x, y z, y^{2}-z^{2}+x y\right)$ & 1 \\
$\left(x, y, z^{3}+\left(y^{2}+x^{2}\right) z\right)$ & 1 \\
$\left(x, y, z^{3}+\left(y^{2}-x^{2}\right) z\right)$ & 1 \\
$\left(x, y, z^{3}-\left(y^{2}-x^{2}\right) z\right)$ & 1 \\
$\left(x, y, z^{3}-\left(y^{2}+x^{2}\right) z\right)$ & 1 \\
$\left(x, y, y z+z^{4}+x^{2} z^{2}\right)$ & 1 \\
$\left(x, y, y z+z^{4}-x^{2} z^{2}\right)$ & 1 \\
$\left(x, y, y z+x z^{2}+z^{5}\right)$ & 1 \\
\hline
\end{tabular}

Table 9: Monogerms for 3-dof spatial motions of codimension $\leq 1$.

germs of higher modality whose versal unfoldings are as yet unstudied. It is probably impractical to continue this listing process very much further. The evidence is that the motions which arise in engineering robotics have considerable extra structure, limiting the range of trajectory germs which can appear, and suggesting new lines of development.

\section{References}

[1] Arnold, V. I. (1976) Wavefront Evolution and Equivariant Morse Lemma. Comm. Pure Appl. Math. 29 557-582.

[2] Arnold, V. I. (1979) Indices of Singular points of 1-Forms on a Manifold with Boundary, Convolution of Invariants of Reflection Groups, and Singular Projections of Smooth Surfaces. Russian Math. Surveys, 34(2), 3-38.

[3] Ball, R. S. (1900) The Theory of Screws, Cambridge University Press.

[4] Bennett, G. T. (1903) A New Mechanism. Engineering, 76, 777-778.

[5] Bennett, G. T. (1913) The Skew Isogram Mechanism. Proc. London Math. Soc. (2nd series), 13, 151-173.

[6] Bottema, O. and Roth, B. (1990) Theoretical Kinematics. Dover Publications.

[7] Bruce, J. W. (1978) Simple Singularities of Mappings $(\mathbf{C}, 0) \rightarrow\left(\mathbf{C}^{2}, 0\right)$. Preprint, University of Liverpool.

[8] Bruce, J. W. (1986) A Classification of 1-Parameter Families of Map-Germs $\left(\mathbf{R}^{3}, 0\right) \rightarrow\left(\mathbf{R}^{3}, 0\right)$ with Applications to Condensation Problems. Jour. Lond. Math. Soc., 33(2), 375-384.

[9] Bruce, J. W. and Gaffney, T. J. (1982) Simple Singularities of Mappings $(\mathbf{C}, 0) \rightarrow\left(\mathbf{C}^{2}, 0\right)$. J. London Math. Soc., 26, 465-474. 
[10] Bruce, J. W. and du Plessis, A. A. (1995) Complete Transversals, Preprint, University of Liverpool.

[11] Cocke, M. W., Donelan, P. S. and Gibson, C. G. On the Genericity of Motions arising from Planar Linkages. In preparation.

[12] Donelan, P. S. (1988) Generic Properties in Euclidean Kinematics, Acta Applicandae Mathematicae, 12, 265-286.

[13] Donelan, P. S. (1993) On the Geometry of Planar Motions. Quarterly J. of Math. Oxford, 44(2), 165-181.

[14] Donelan, P. S. and Gibson, C. G. (1991) First-Order Invariants of Euclidean Motions. Acta Applicandae Mathematicae, 24, 233-251.

[15] Donelan, P. S. and Gibson, C. G. (1993) On the Hierarchy of Screw Systems. Acta Applicandae Mathematicae, 32, 267-296.

[16] Donelan, P. S. and Gibson, C. G. Instantaneous Singular Sets for Spatial Motions. In preparation.

[17] Donelan, P. S., Gibson, C. G. and Hawes, W. Trajectory Singularities of General Planar Motions. In preparation.

[18] Dorf, R. C. (Ed.) (1988) International Encyclopedia of Robotics, John W. Wiley and Sons.

[19] Gaffney, T., du Plessis, A. A. and Wilson, L. C. (1990) Map-Germs Determined by their Discriminant. To appear in the Proceedings of the Hawaii-Provence Singularities Conferences, Travaux en Cours, Hermann, Paris.

[20] Gaffney, T. (1983) The Structure of $T \mathcal{A}(f)$, Classification and an Application to Differential Geometry. AMS Proceedings of Symposia in Pure Mathematics, Singularities, Part 1, 40, 409-427.

[21] Gibson, C. G. (1992) Kinematic Singularities - A New Mathematical Tool. Third International Workshop on Advances in Robot Kinematics, Ferrara, Italy, 209-215.

[22] Gibson, C. G., Hawes, W. H. and Hobbs, C. A. (1994) Local Pictures for General TwoParameter Motions of the Plane. In Advances in Robot Kinematics and Computational Geometry, Kluwer Academic Publishers, 49-58.

[23] Gibson, C. G. \& Hobbs, C. A. (1995) Local Models for General One-Parameter Motions of the Plane and Space. Proc. Royal Society of Edinburgh, 125A, 639-656.

[24] Gibson, C. G. and Hobbs, C. A. (1996) Singularity and Bifurcation for General TwoDimensional Planar Motions. To appear in New Zealand J. Math.

[25] Gibson, C. G. and Hobbs, C. A. (1992) Simple Singularities of Space Curves. Math. Proc. Camb. Phil. Soc., 113, 297-310.

[26] Gibson, C. G., Hobbs, C. A. \& Marar, W. L. (1996) On Versal Unfoldings of Singularities for General Two-Dimensional Spatial Motions. To appear in Acta Applicandae Mathematicae.

[27] Gibson, C. G. and Hunt, K. H. (1992) Geometry of Screw Systems. Mech. Machine Theory, $12,1-27$. 
[28] Gibson, C. G., Marsh, D. and Xiang, Y. (1996) An Algorithm to Generate the Transition Curve of the Planar Four-bar Mechanism. Mech. Machine Theory, 31, 381-395.

[29] Gibson, C. G., Marsh, D. and Xiang, Y. Singular Aspects of Generic Planar Motions with Two Degrees of Freedom. Preprint, University of Liverpool.

[30] Gibson, C. G. and Newstead, P. E. (1986) On the Geometry of the Planar 4-Bar Mechanism. Acta Applicandae Mathematicae, 7, 113-135.

[31] Hain, K. (1964) Das Spektrum des Gelenkvierecks bei veränderlicher Gestell-Länge. VDI Forsch Ing.-Wes., 30, 33-42.

[32] Hawes, W. (1995) Multi-Dimensional Motions of the Plane and Space. Ph. D. Thesis, University of Liverpool.

[33] Hobbs, C. A. (1993) Kinematic Singularities of Low Dimension. Ph. D. Thesis, University of Liverpool.

[34] Hobbs, C. A. and Kirk, N. P. (1996) On the Classification and Bifurcation of Multigerms of Maps from Surfaces to 3-Space. Preprint, Oxford-Brookes University.

[35] Hunt, K. H. (1978) Kinematic Geometry of Mechanisms, Clarendon Press, Oxford.

[36] Jessop, C. M.(1916) Quartic Surfaces with Singular Points, Cambridge University Press.

[37] Kirk, N. P. (1993) Computational Aspects of Singularity Theory. Ph. D. Thesis, University of Liverpool.

[38] Klein, F. (1871) Notiz Betreffend dem Zusammenhang der Liniengeometrie mit der Mechanik starrer Körper. Math. Ann., 4, 403-415.

[39] Marar, W. L. and Tari, F. (1995) On the Geometry of Simple Germs of Corank 1 Maps from $\mathbf{R}^{3}$ to $\mathbf{R}^{3}$. Preprint, University of Liverpool.

[40] Martinet, J. (1982) Singularities of Smooth Functions and Maps. London Mathematical Society Lecture Note Series, 58, Cambridge University Press.

[41] Mather, J. N. (1970) Stability of $\mathrm{C}^{\infty}$ Mappings: VI The Nice Dimensions. Springer Lecture Notes in Mathematics, Proceedings of Liverpool Singularities Symposium I, 192, 207-253.

[42] Mond, D. M. Q. (1985) On the Classification of Germs of Maps from $\mathbf{R}^{2}$ to $\mathbf{R}^{3}$. Proc. London Math. Soc., 50(3), 333-369.

[43] Montaldi, J. A. (1991) On Generic Composites of Maps. Bull. London Math. Soc., 23, 81-85.

[44] Morin, B. (1965) Formes Canoniques des Singularités d'une Application Différentiable. Compt. Rendus Acad. Sci. Paris, 260, 5662-5665, 6503-6506.

[45] Müller R. (1889) Über die Doppelpunkte der Koppelkurve Z. Math. Phys., 34, 303-305 \& 372375, [trans. Tesar D. in (Translations of) Papers on Geometrical Theory of Motion applied to Approximate Straight Line Motion, Kansas State University Bulletin, 46 (1962), Special Report No. 21.]

[46] Müller R. (1903) Über einige Kurven, die mit der Theorie des ebenen Gelekvierecks in Zusammenhang stehen Z. Math. Phys., 48, 224-248, [trans. Tesar D. in (Translations of) Papers on Geometrical Theory of Motion applied to Approximate Straight Line Motion, Kansas State University Bulletin, 46 (1962), Special Report No. 21.] 
[47] Niemann, S. H. (1978) Geometry and Mechanics. Ph.D. Thesis, Oxford University.

[48] Pai D. K. and Leu M. C. (1992) Genericity and Singularities of Robot Manipulators. IEEE Trans. Robotics and Automation, 8, 545-559.

[49] du Plessis, A. A. (1980) On the Determinacy of Smooth Map-Germs. Invent. Math., 58, $107-160$.

[50] Rieger, J. H. and Ruas, M. A. S. (1987) Classification of $\mathcal{A}$-simple germs from $k^{n}$ to $k^{2}$. Comp. Math., 79, 99-108.

[51] Robertson, G. D. and Torfason, L. E. The Queeroid - A New Kinematic Surface. Proc. Fourth World Congress on the Theory of Machines and Mechanisms, 717-719.

[52] Tchoń, K. and Muszyński, R. (1995) Singularities of Non-Redundant Robot Kinematics. To appear in Int. J. Robotics Research.

[53] Thom, R. (1975) Structural Stability and Morphogenesis, W. A. Benjamin, Inc. Reading, Massachusetts.

[54] Wall, C. T. C. (1980) Affine Cubic Functions III. The Real Plane. Math. Proc. Camb. Phil. Soc., 87(1), 1-14.

[55] West, J. M. (1995) The Differential Geometry of the Cross Cap. Ph.D. Thesis, University of Liverpool.

[56] Whitney, H. (1955) On Singularities of Mappings of Euclidean Space I: Mappings of the Plane to the Plane. Ann. Math., 62, 374-410.

[57] Wilkinson, T. (1991) The Geometry of Folding Maps, Ph.D. Thesis, University of Newcastle.

[58] Xiang, Y. (1995) Bifurcation of Singularities of Planar Mechanisms with One- or Two-Degrees of Freedom. Ph.D. Thesis, Napier University.

\author{
P. S. Donelan \\ Mathematics \\ Victoria University of Wellington \\ Private Bag, Wellington \\ New Zealand. \\ e-mail: peter.donelan@vuw.ac.nz
}

C. G. Gibson

Mathematical Sciences

University of Liverpool

PO Box 147, Liverpool L69 3BX

United Kingdom.

e-mail: c.g.gibson@liverpool.ac.uk 